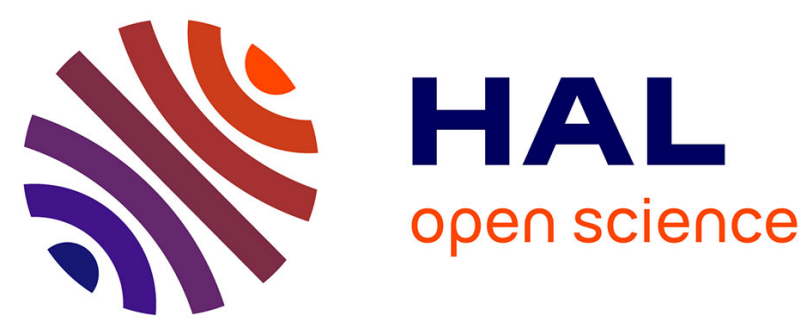

\title{
Delivery Delay Analysis for Roadside Unit Deployment in Vehicular Ad Hoc Networks with Intermittent Connectivity
}

Yu Wang, Jun Zheng, Nathalie Mitton

\section{- To cite this version:}

Yu Wang, Jun Zheng, Nathalie Mitton. Delivery Delay Analysis for Roadside Unit Deployment in Vehicular Ad Hoc Networks with Intermittent Connectivity. IEEE Transactions on Vehicular Technology, 2016, PP (99), 10.1109/TVT.2015.2506599 • hal-01233732

HAL Id: hal-01233732

https://hal.inria.fr/hal-01233732

Submitted on 8 Mar 2016

HAL is a multi-disciplinary open access archive for the deposit and dissemination of scientific research documents, whether they are published or not. The documents may come from teaching and research institutions in France or abroad, or from public or private research centers.
L'archive ouverte pluridisciplinaire HAL, est destinée au dépôt et à la diffusion de documents scientifiques de niveau recherche, publiés ou non, émanant des établissements d'enseignement et de recherche français ou étrangers, des laboratoires publics ou privés. 


\title{
Delivery Delay Analysis for Roadside Unit Deployment \\ in Vehicular Ad Hoc Networks with Intermittent Connectivity
}

\author{
Yu Wang ${ }^{1}$, Jun Zheng ${ }^{1 *}$, and Nathalie Mitton ${ }^{2}$ \\ ${ }^{1}$ National Mobile Communications Research Laboratory \\ Southeast University \\ 2 Si Pai Lou, Nanjing, Jiangsu 210096, China \\ ${ }^{2}$ Inria Lille-Nord Europe \\ 40 Avenue Halley, 59650 Villeneuve D’Ascq, France \\ *Corresponding author: Jun Zheng (junzheng@seu.edu.cn)
}

\begin{abstract}
This paper studies the information delivery delay analysis for roadside unit deployment in a vehicular ad hoc network (VANET) with intermittent connectivity. A mathematical model is developed to describe the relationship between the average delay for delivering road condition information and the distance between two neighbor RSUs deployed along a road. The derived mathematical model considers a straight highway scenario where two RSUs are deployed at a distance without any direct connection and vehicles are sparsely distributed on the road with road condition information randomly generated between the two neighbor RSUs. Moreover, the model takes into account the vehicle speed, the vehicle density, the likelihood of an incident, and the distance between two RSUs. The effectiveness of the derived mathematical model is verified through simulation results. Given the information delivery delay constraint of a time-critical application, this model can be used to estimate the maximum distance allowed between two neighbor RSUs, which can provide a reference for the deployment of RSUs in such scenarios.
\end{abstract}

Keywords - delay analysis; information delivery; roadside unit; VANET

\section{I . Introduction}

Vehicular Ad Hoc Networks (VANETs) promise a variety of potential applications in providing road safety and in-vehicle entertainment services. One of the important VANET applications is to collect road condition information via vehicles and disseminate the collected information to all vehicles in the network in order to help avoid traffic jams, reduce car accidents, and save fuel consumption. Due to its highly dynamic characteristic, a VANET is often of intermittent connectivity, which would increase the delay for disseminating collected road condition information and thus affect the quality of service (QoS) provisioned to users. To address this problem, roadside units (RSUs) can be deployed in a VANET to improve the network connectivity, and help disseminate 
road condition information. Obviously, the more densely the RSUs are deployed, the better the network connectivity can be obtained. However, deploying RSUs is costly. It is impractical to deploy RSUs in a dense scale, in particular, in those remote areas. This means that two neighbor RSUs can be deployed along a road without any direct connection, which would increase the delay for disseminating road condition information collected outside the coverage of an RSU. For many applications, however, road condition information is time critical. It is important to deliver such time-critical information to an RSU within a delay constraint so that the RSU can help timely disseminate the information to the vehicles passing through it. To this end, two neighbor RSUs should not be deployed at a distance larger than a certain value. Therefore, it is helpful to find out and analyze the relationship between the information delivery delay and the distance between two neighbor RSUs deployed along a road, which is the motivation for this work.

In this paper, we study the information delivery delay analysis for roadside unit deployment in a VANET with intermittent connectivity. We derive a mathematical model to describe the relationship between the average delay for delivering road condition information and the distance between two neighbor RSUs deployed along a road. We consider a straight highway scenario where two RSUs are deployed at a distance without any direct connection and vehicles are sparsely distributed on the road with road condition information randomly generated between the two neighbor RSUs. Moreover, we take into account the vehicle speed, the vehicle density, the likelihood of an incident, and the distance between two RSUs in the mathematical model. Simulation experiments are conducted to verify the effectiveness of the derived mathematical model and investigate the impacts of different parameters on the average information delivery delay.

The remainder of the paper is organized as follows. Section II reviews related work in the literature. Section III derives the mathematical model and analyzes the information delivery delay. Section IV presents simulation results to verify the effectiveness of the mathematical model. Section V concludes this paper.

\section{II . Related work}

Delay analysis for sparse VANETs has been studied in the literature [1-8]. In [1-4], Khabbaz et al. proposed several information release mechanisms to achieve a delay-minimal information delivery in the context of an intermittent roadside network. However, all of them considered how to send the information to passing vehicles, rather than send the information to RSUs by passing vehicles. In [5], Abdrabou et al. studied the multi-hop packet delivery delay in a low-density network scenario, where an end-to-end path is not available between a vehicle and the nearest RSU, in order to obtain the maximum distance between RSUs that stochastically limits the worst-case packet delivery delay to a certain bound. However, this work only considers the scenario where vehicles move in 
one direction. In [6], Abdrabou et al. presented a mathematical model for calculating the message delivery delay distribution on a two-lane road, where vehicles in one direction can carry messages for the vehicles in the other direction and have the freedom to leave the road from randomly distributed road junctions with a certain probability. However, they considered the infrastructure-based RSUs, where the RSUs are connected through fiber or broadband wireless links. In [7], Wisitpongphan et al. proposed a model for analyzing the packet delivery delay between disconnected vehicles. Based on [7], Reis et al. proposed a model for analyzing the average time taken to propagate a packet to disconnected nodes when considering both scenarios of connected and disconnected RSUs in [8]. The results show significant delay improvement with RSU deployment, particularly in multi-cluster communication scenarios. However, both of them only considered the information delivery delay from vehicle to vehicle without considering the delivery delay between a vehicle and an RSU. To the best of our knowledge, there is no work found in the literature that studies the delay analysis of delivering road condition information to non-connected RSUs by passing vehicles for RSU deployment.

\section{Modeling and Analysis of Delivery Delay}

In this section, we derive a mathematical model to describe the relationship between the information delivery delay and the distance between two neighbor RSUs deployed along a road.

1. Network Scenario and Problem Description

We consider a straight road segment between two neighbor RSUs on a sparse highway with two lanes in opposite directions, and low connectivity and traffic load, as illustrated in Fig. 1. The distance between RSU1 and RSU2 is denoted by $d$. The communication radius of each RSU is denoted by $R_{u}$. The communication radius of each vehicle is denoted by $R_{v}$. We assume that $R_{u} \geq R_{v}$. The two neighbor RSUs are deployed without any direct connection, i.e., $d>>2 R_{u}$. The inter-vehicle spacing of westbound vehicles and eastbound vehicles are exponentially distributed with $\lambda_{e}$ and $\lambda_{w}$ [7], respectively, where $\lambda_{e}$ and $\lambda_{w}$ are the average arrival rates of the westbound vehicles and eastbound vehicles, respectively. All vehicles moving in one direction have the same speed with $v_{e}$ and $v_{w}$ as the speeds for the eastbound vehicles and westbound vehicles, respectively. The vehicles that move in the same direction and can communicate with one another in one hop or multiple hops form a cluster, as shown in Fig. 2. As all vehicles moving in one direction have the same speed, all clusters in the same direction will keep their respective spacing and thus cannot communicate with each other. An incident occurs randomly at a location with a distance $x$ from RSU1, i.e., $x[0, d]$. Once an incident occurs, the closest vehicle to the occurring location of the incident will first arrive at the location and collect the incident information. This vehicle is referred to as a source node, which will deliver the collected information to the two neighbor RSUs directly or indirectly 
via other vehicles. A vehicle that can forward the collected information to an RSU is referred to as a forwarding node. We define the average information delivery delay as the average time from the instant when an incident occurs to the instant when both the RSUs have received the incident information. Since this delay depends on the distance between the two neighbor RSUs, it is interesting to find out the relationship between the distance and the delay, which would be helpful to RSU deployment in order to meet the delay constraint of an application. Therefore, the problem considered in this paper is to develop a mathematical model that describes the average information delivery delay and the distance between two neighbor RSUs deployed along a straight highway.

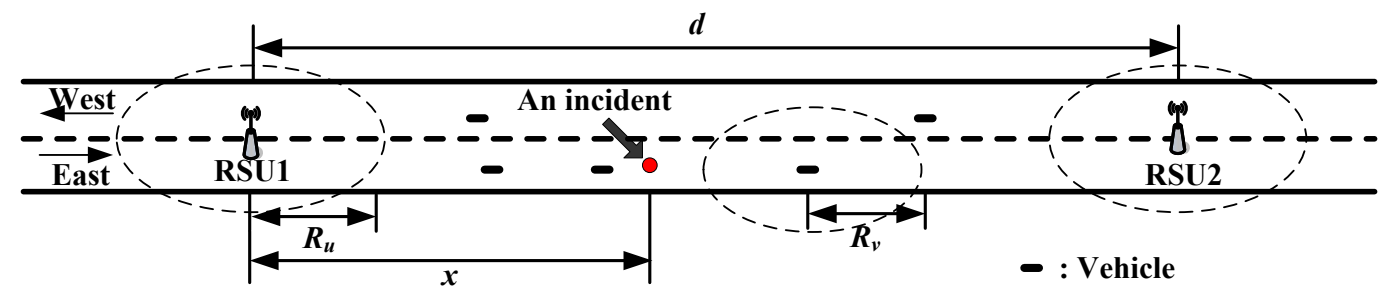

Fig.1 Basic network scenario.

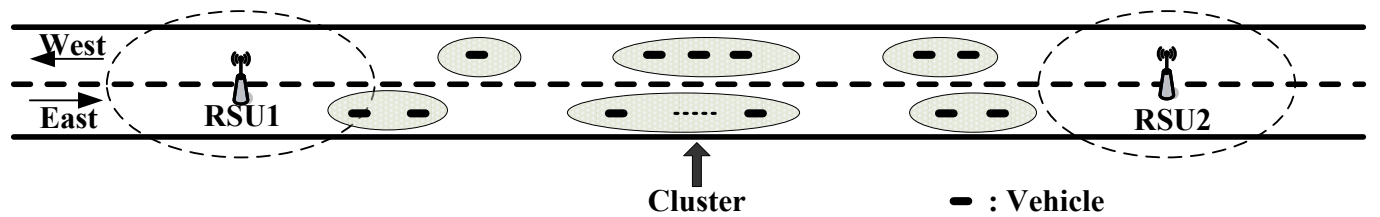

Fig.2 Vehicle clusters on a road.

2. Modeling and Analysis of Delivery Delay

Considering the basic network scenario shown in Fig. 1, the average information delivery delay can be calculated as

$$
T=T_{0}+T_{S},
$$

where $T$ is the average delivery delay, $T_{0}$ is the average time it takes for the closest vehicle to the occurring location of an incident (i.e., the source node) to arrive at the occurring location of the incident, and $T_{S}$ is the average time it takes for the source node to deliver the collected information from the occurring location of an incident to an RSU. Since we are considering a sparse highway scenario, both the network connectivity and the traffic load are low. In this case, the incident information will be delivered from the source node to the destination node via one or multiple vehicles in a "store-carry-forward" manner, and the delivery delay is typically on the order of seconds or even minutes [7]. For this reason, if the destination node of the collected information is within the transmission radius of the source node, the delay caused by direct transmission between the two nodes would be much smaller than the delivery delay caused by "store-carry-forward". Therefore, we will mainly consider the 
delivery delay caused by "store-carry-forward" and ignore the direct transmission delay between a couple of source and destination nodes in the analytical model.

Let $f(x)$ denote the probability density function of the distance $x$. The expectation of $x$ can be calculated as follows

$$
E[X]=\int_{0}^{d} x \cdot f(x) d x, f(x)(0 \leq x \leq d) .
$$

As explained earlier, $T_{S}$ is the average time for the collected information to be delivered from the occurring location of an incident to an RSU. Obviously, $T_{S}$ depends on the distance between the two neighbor RSUs. When a source node has collected the incident information, it will deliver the collected information to both RSU1 and RSU2. Since it takes different time for the source node to deliver the information to RSU1 and RSU2, respectively, we take the longer one in calculating $T_{S}$ because only after $T_{S}$ both RSU1 and RSU2 can receive the information. Therefore, we have

$$
T_{S}=\int_{0}^{d} \max \left\{T_{R S U 1}, T_{R S U 2}\right\} \cdot f(x) d x,
$$

where $T_{R S U 1}$ and $T_{R S U 2}$ represents the delay for the collected information to be delivered from the occurring location of the incident to RSU1 and RSU2, respectively.

Next we analyze the average information delivery delay $T$ in the following three cases:

- Case 2.1: The incident occurs at a location where $x \in\left[0, R_{v}\right]$;

- Case 2.2: The incident occurs at a location where $x \in\left[R_{v}, d-R_{v}\right]$;

- Case 2.3: The incident occurs at a location where $x \in\left[d-R_{v}, d\right]$.

Without loss of generality, we assume that when an incident occurs, an eastbound vehicle is the closest to the occurring location of the incident and will first arrive at the occurring location of the incident. This vehicle is called source node hereafter. The notations used in the analysis are defined as follows:

$n_{e}$ : The size of an eastbound cluster, i.e., the number of vehicles in an eastbound cluster;

$n_{w}$ : The size of a westbound cluster, i.e., the number of vehicles in a westbound cluster;

$l_{e}$ : The length of an eastbound cluster, i.e., the distance between the first vehicle and the last vehicle in an eastbound cluster;

$l_{w}$ : The length of a westbound cluster, i.e., the distance between the first vehicle and the last vehicle in a westbound cluster;

$E\left[l_{e}\right]$ : The average length of an eastbound cluster;

$E\left[l_{w}\right]$ : The average length of a westbound cluster; 
$f_{e}\left(n_{e}\right)$ : The probability mass function $(\mathrm{PMF})$ of $N_{e}$;

$f_{w}\left(n_{w}\right)$ : The probability mass function (PMF) of $N_{w}$;

$E\left[d_{v}^{e}\right]:$ The average distance between two vehicles within an eastbound cluster;

$E\left[d_{v}^{w}\right]$ : The average distance between two vehicles within a westbound cluster;

$E\left[d_{c}^{e}\right]$ : The average distance between two neighbor eastbound clusters, i.e., the expected distance between the last vehicle of an eastbound cluster and the first vehicle of the following eastbound cluster;

$E\left[d_{c}^{w}\right]$ : The average distance between two neighbor westbound clusters, i.e., the expected distance between the last vehicle of a westbound cluster and the first vehicle of the following westbound cluster.

2.1 Case 2.1: The incident occurs at $x \in\left[0, R_{v}\right]$

In Case 2.1, the incident occurs within the communication radius of RSU1. After the source node detects the incident, it can transmit the information to RSU1 directly. Thus, the time it takes for the source node to directly forward the incident information to RSU1 can be ignored. Let $f_{1}(x)$ denote the conditional probability density function of the distance $x$ in $\left[0, R_{v}\right]$. Then, the average information delivery delay can be written as follows

$$
T_{1}=T_{01}+T_{S 1},
$$

where $T_{01}$ is the average time it takes for the vehicle closest to the occurring location of an incident (i.e., the source node) to arrive at the occurring location of the incident, and $T_{S 1}$ is the average time it takes for the source node to deliver the collected information from the occurring location of the incident to RSU2. Next we analyze $T_{01}$ and $T_{S 1}$, respectively.

\subsubsection{Analysis of $T_{01}$}

According to the property of an exponential distribution, the average time that it takes for the eastern vehicle node to arrive at the left end point of interval $\left[0, R_{v}\right]$ is $1 /\left(\lambda_{e} v_{e}\right)$. Thus, $T_{01}$ can be calculated as

$$
T_{01}=1 / v_{e} \cdot \int_{0}^{R_{v}} x \cdot f_{1}(x) d x+1 /\left(\lambda_{e} v_{e}\right) .
$$

\subsubsection{Analysis of $T_{S 1}$}

Like [7], we assume that the source node is statistically located in the center of its cluster. As we assumed that the source node is an eastbound vehicle, the forwarding direction of the incident information to RSU2 is the same as the moving direction of the source node. Since the direct transmission delay can be ignored, the incident information can be delivered to the first vehicle in the cluster without any delay. In this case, the first vehicle 
becomes a forwarding node, which is denoted by node 1, as shown in Fig. 3. If node 1 is in the communication radius of RSU2, the information can be transmitted to RSU2 directly. Thus, we only need to consider the case where node 1 is out of the communication radius of RSU2. This case happens if the length of the eastbound cluster satisfies the following condition:

$$
l_{e} / 2-R_{v}<d-x-R_{u},
$$

and the probability that this case happens is given by

$$
p_{212}=\operatorname{Pr}\left\{l_{e} / 2-R_{v}<d-x-R_{u}\right\} .
$$

According to [7], the average distance between two vehicles in an eastbound cluster can be calculated as

$$
E\left[d_{v}^{e}\right]=1 / \lambda_{e}-R_{v} \cdot e^{-\lambda_{e} R_{v}} /\left(1-e^{-\lambda_{e} R_{v}}\right)
$$

and the PMF of $n_{e}$ is

$$
f_{e}\left(n_{e}\right)=e^{-\lambda_{e} R_{v}}\left(1-e^{-\lambda_{e} R_{v}}\right)^{n_{e}-1} .
$$

Obviously, given $E\left[d_{v}^{e}\right]$ and $n_{e}$, we can obtain

$$
p_{212}=\operatorname{Pr}\left\{l_{e} / 2-R_{v}<d-x-R_{u}\right\}=1-\left(1-e^{-\lambda_{e} R_{v}}\right)^{k_{212}},
$$

where $k_{212}=2\left(d-x-R_{u}+R_{v}\right) / E\left[d_{v}^{e}\right]$.

Let $E_{212}$ denote the conditional expectation of $l_{e} / 2$ under condition $l_{e} / 2<d-x-R_{u}+R_{v}$. We can obtain

$$
E_{212}=E\left[l_{e} / 2 \mid l_{e} / 2<d-x-R_{u}+R_{v}\right]=0.5 E\left[d_{v}^{e}\right] E\left[n_{e} \mid n_{e}<k_{212}\right]
$$

where

$$
E\left[n_{e} \mid n_{e}<k_{212}\right]=\left[1-\left(k_{212}+1\right)\left(1-e^{-\lambda_{e} R_{v}}\right)^{k_{212}}+k_{212}\left(1-e^{-\lambda_{e} R_{v}}\right)^{k_{212}+1}\right] /\left\{\left[1-\left(1-e^{-\lambda_{e} R_{v}}\right)^{k_{212}}\right] e^{-\lambda_{e} R_{v}}\right\} .
$$

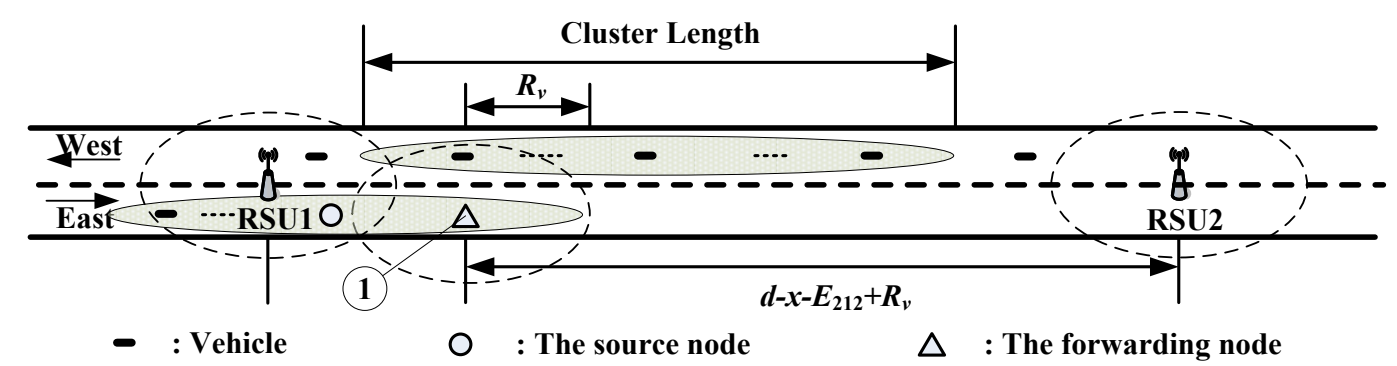

Fig.3 The westbound cluster is out of the communication radius of RSU2.

Further, this case can be divided into the following two sub-cases:

- Case 2.1.2.0: There are one or more westbound vehicles in the coverage of node 1 .

- Case 2.1.2.1: There is no westbound vehicle in the coverage of node 1 . 
Case 2.1.2.0

Case 2.1.2.0 happens when there is at least one westbound vehicle within the communication radius of node 1 . Let $p_{2120}$ denote the probability that Case 2.1.2.0 happens. Thus, we have

$$
p_{2120}=1-e^{-2 \lambda_{w} R_{v}} .
$$

In this case, if the last vehicle of the westbound cluster is within the communication radius of RSU2, the information can be transmitted to RSU2 directly and the direct transmission delay can be ignored. However, if the last vehicle of the westbound cluster is out of the communication radius of RSU2, i.e., the length of the westbound cluster satisfies the following condition:

$$
l_{w} / 2<d-x-E_{212}+R_{v},
$$

information will be forwarded to RSU2 by node 1 . The probability of this case is given by

$$
p_{21200}=\operatorname{Pr}\left\{l_{w} / 2<d-x-E_{212}+R_{v}\right\} .
$$

According to [7], the average distance between two vehicles in a westbound cluster can be calculated as

$$
E\left[d_{v}^{w}\right]=1 / \lambda_{w}-R_{v} \cdot e^{-\lambda_{w} R_{v}} /\left(1-e^{-\lambda_{w} R_{v}}\right),
$$

and the PMF of $n_{w}$ is

$$
f_{w}\left(n_{w}\right)=e^{-\lambda_{w} R_{v}}\left(1-e^{-\lambda_{w} R_{v}}\right)^{n_{w}-1} .
$$

Obviously, given $E\left[d_{v}^{w}\right]$ and $n_{w}$, we can obtain

$$
p_{21200}=\operatorname{Pr}\left\{l_{w} / 2<d-x-E_{212}+R_{v}\right\}=1-\left(1-e^{-\lambda_{w} R_{v}}\right)^{k_{21200}},
$$

where $k_{21200}=2\left(d-x-E_{212}+R_{v}\right) / E\left[d_{v}^{w}\right]$. Therefore, the delivery delay can be calculated as

$$
T_{21200}=\left(d-x-R_{u}-E_{212}+R_{v}\right) / v_{e} .
$$

Case 2.1.2.1

Case 2.1.2.1 happens when there is no westbound vehicle within the communication radius of node 3. The probability that Case 2.1.2.1 happens can be calculated as

$$
p_{2121}=1-p_{2120} .
$$

In this case, the information will be forwarded to RSU2 by node 1 . Thus, the delivery delay $T_{2121}$ equals to $T_{21200}$.

Considering all the above cases, we have

$$
T_{S 1}=p_{212} \cdot\left(p_{2120} \cdot p_{21200} \cdot T_{21200}+p_{2121} \cdot T_{2121}\right) .
$$

2.2 Case 2.2: The incident occurs at $x \in\left[R_{v}, d-R_{v}\right]$

Let $f_{2}(x)$ denote the conditional probability density function of the distance $x$ in $\left[R_{v}, d-R_{v}\right]$. The average 
information delivery delay can be given as

$$
T_{2}=T_{02}+T_{S 2}
$$

where $T_{02}$ is the average time that it takes for the source node to arrive at the occurring location of the incident, and $T_{S 2}$ is the average time it takes for the source node to deliver the collected information from the occurring location of the incident to both of the neighboring RSUs. Since it takes a different time to deliver the information to RSU1 and RSU2, we need to calculate $T_{R S U 1}$ and $T_{R S U 2}$, and take the longer one in calculating $T_{S 2}$. Next we analyze $T_{02}$ and $T_{S 2}$, respectively.

\subsubsection{Analysis of $T_{02}$}

According to the property of an exponential distribution, the average inter-vehicle spacing of eastbound vehicles is $1 / \lambda_{e}$. Let $y$ denote the distance between RSU1 and the vehicle closest to the occurring location of an incident. Obviously, we have $x-1 / \lambda_{e} \leq y \leq x$, as illustrated in Fig. 4. Let $f_{2}^{\prime}(x)$ denote the conditional probability density function of the distance $x$ in $\left[y, y+1 / \lambda_{e}\right]$. Thus, the average time that it takes for the closest vehicle to the occurring location of an incident to arrive at the occurring location of the incident can be calculated as

$$
T_{02}=\left[\int_{y}^{y+1 / \lambda_{e}} x \cdot f_{2}^{\prime}(x) d x-y\right] / v_{e}
$$

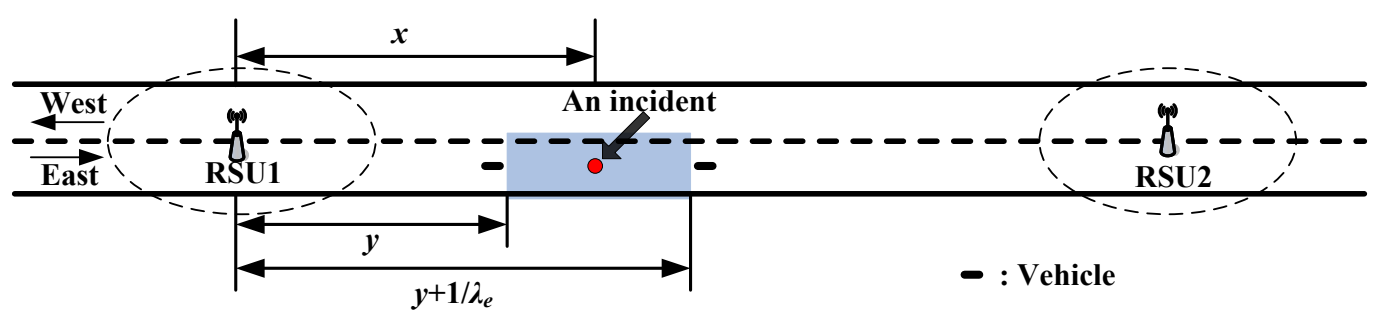

Fig.4 Relative position of the source node and the location of an incident.

\subsubsection{Analysis of $T_{S 2}$}

As discussed earlier, $T_{S 2}$ is the longest one in $T_{R S U 1}$ and $T_{R S U 2}$. Next we first analyze $T_{R S U 1}$ and $T_{R S U 2}$, respectively.

\subsubsection{Analysis of $T_{R S U 1}$}

Like [7], we assume that the source node is statistically located in the center of its cluster, as shown in Fig. 5. As we assumed that the source node is an eastbound vehicle, the delivery direction of the incident information to RSU1 is opposite to the moving direction of the source node. Since the direct transmission delay is ignored, the incident information can be delivered to the last vehicle in the cluster without any delay. In this case, this vehicle becomes a forwarding node, which is denoted by node 2 in Fig. 5 . 


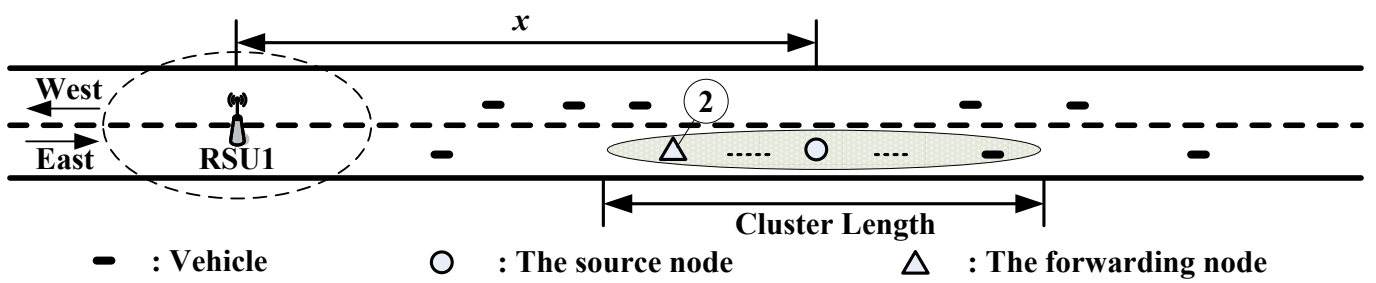

Fig.5 The forwarding node is out of the communication radius of RSU1.

If node 2 is within the communication radius of RSU1, the information can be directly transmitted to RSU1. Since the direct transmission delay can be ignored, we only need to analyze the case where node 2 is out of the communication radius of RSU1. In this case, the forwarding node can take advantage of the westbound vehicles to forward the information to RSU1.

This case happens if the length of the eastbound cluster satisfies the following condition:

$$
l_{e} / 2-R_{v}<x-R_{u},
$$

and the probability that this case happens is given by

$$
p_{2221}=\operatorname{Pr}\left\{l_{e} / 2-R_{v}<x-R_{u}\right\} .
$$

Similar to Eq. (9), we can obtain

$$
p_{2221}=\operatorname{Pr}\left\{l_{e} / 2-R_{v}<x-R_{u}\right\}=1-\left(1-e^{-\lambda_{e} R_{v}}\right)^{k_{2221}},
$$

where $k_{2221}=2\left(x-R_{u}+R_{v}\right) / E\left[d_{v}^{e}\right]$.

Let $E_{2221}$ denote the conditional expectation of $l_{e} / 2$ under condition $l_{e} / 2-R_{v}<x-R_{u}$. We can obtain

$$
\begin{aligned}
E_{2221} & =E\left[l_{e} / 2 \mid l_{e} / 2<x-R_{u}+R_{v}\right] \\
& =0.5 E\left[d_{v}^{e}\right] \cdot\left[1-\left(1-e^{-\lambda_{e} R_{v}}\right)^{k_{2221}} \cdot\left(k_{2221} e^{-\lambda_{e} R_{v}}+1\right)\right] /\left\{\left[1-\left(1-e^{-\lambda_{e} R_{v}}\right)^{k_{2221}}\right] e^{-\lambda_{e} R_{v}}\right\}^{.}
\end{aligned}
$$

Further, we divide this case into two sub-cases:

- Case 2.2.2.1.0: There are one or more westbound vehicles within the coverage of node 2 .

- Case 2.2.2.1.1: There is no westbound vehicle in the coverage of node 2.

Case 2.2.2.1.0

Case 2.2.2.1.0 happens when there is at least one westbound vehicle within the communication radius of node 2. Let $p_{22210}$ denote the probability that Case 2.2.2.1.0 happens. Thus, we have

$$
p_{22210}=1-e^{-2 \lambda_{w} R_{v}} .
$$

In this case, the first vehicle of the corresponding westbound cluster becomes a forwarding node, which is denoted by node 3, as shown in Fig. 6. If node 3 is within the communication radius of RSU1, the information can be directly transmitted to RSU1 and the direct transmission delay can be ignored. Thus, we only need to analyze that 
the case where node 3 is out of the communication radius of RSU1, i.e., the length of the westbound cluster satisfies the following condition:

$$
l_{w} / 2<x-E_{2221}+R_{v} .
$$

In this case, node 3 will store and carry the information until it enters the communication radius of RSU1, when it will transmit the information to RSU1 directly.

Let $p_{222100}$ denote the probability that this case happens. Thus, we have

$$
p_{222100}=\operatorname{Pr}\left\{l_{w} / 2<x-E_{2221}+R_{v}\right\} .
$$

Similar to Eq.(15), we can obtain

$$
p_{222100}=\operatorname{Pr}\left\{l_{w} / 2<x-E_{2221}+R_{v}\right\}=1-\left(1-e^{-\lambda_{w} R_{v}}\right)^{k_{221100}},
$$

where $k_{222100}=2\left(x-E_{2221}+R_{v}\right) / E\left[d_{v}^{w}\right]$.

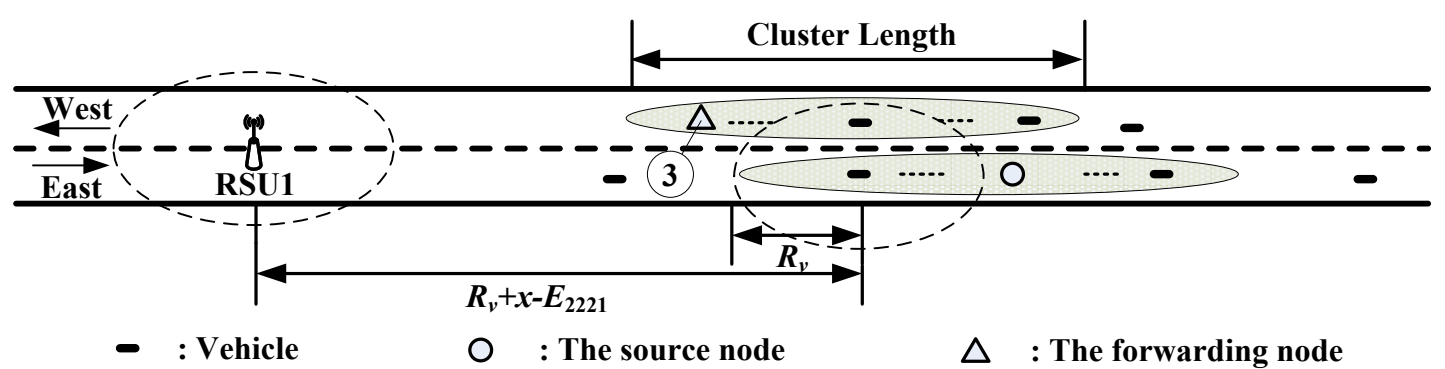

Fig.6 The westbound cluster is out of the communication radius of RSU1.

Therefore, the delivery delay for node 3 to forward the incident information to RSU1 can be approximately calculated as

$$
T_{222100}=\left(x-R_{u}-E_{2221}+2 R_{v}-0.5 E\left[l_{w} / 2 \mid l_{w} / 2<x+R_{v}-E_{2221}\right]\right) / v_{w},
$$

where

$$
\begin{aligned}
& E\left[l_{w} / 2 \mid l_{w} / 2<x+R_{v}-E_{2221}\right] \\
& =0.5 E\left[d_{v}^{w}\right] \cdot\left[1-\left(1-e^{-\lambda_{w} R_{v}}\right)^{k_{222100}} \cdot\left(k_{222100} e^{-\lambda_{w} R_{v}}+1\right)\right] /\left\{\left[1-\left(1-e^{-\lambda_{w} R_{v}}\right)^{k_{222100}}\right] e^{-\lambda_{w} R_{v}}\right\}
\end{aligned}
$$

Case 2.2.2.1.1

Case 2.2.2.1.1 happens when there is no westbound vehicle within the communication radius of node 1 . The probability that case 2.2.2.1.1 happens can be calculated as

$$
p_{22211}=1-p_{22210} .
$$

This case can be further divided into the following two sub-cases:

- Case 2.2.2.1.1.0: There is no westbound vehicle within the communication radius of node 2 and there is no westbound vehicle within the communication range of other nodes in the same cluster either, as shown in 
Fig. 7.

- Case 2.2.2.1.1.1: There is no westbound vehicle within the communication radius of node 2 but there is one or more westbound vehicles within the communication range of a node other than node 2 in the same cluster, as shown in Fig. 8.

Next we analyze Case 2.2.2.1.1.0 and Case 2.2.2.1.1.1, respectively.

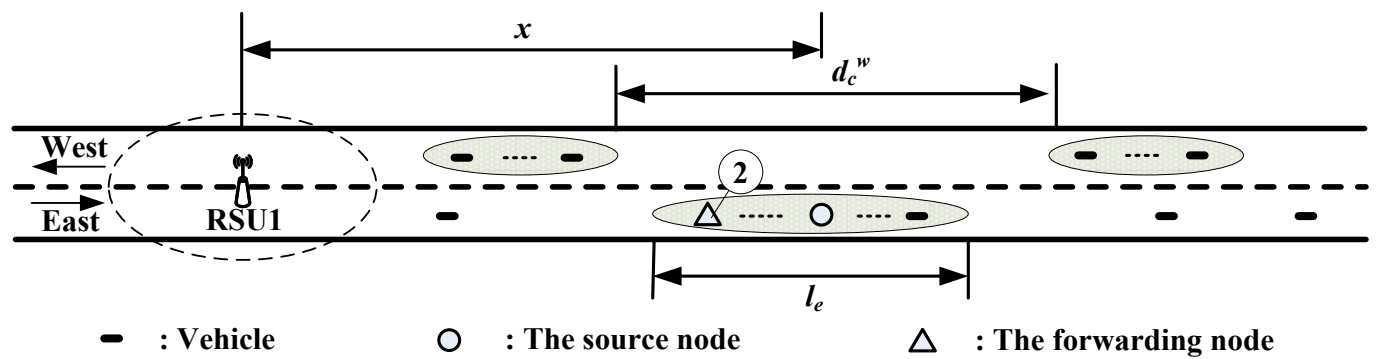

Fig.7 Case 2.2.2.1.1.0.

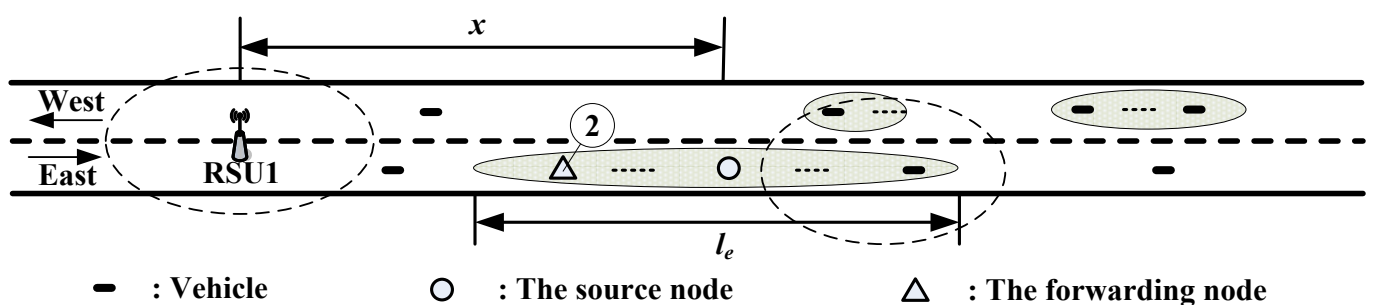

Fig.8 Case 2.2.2.1.1.1.

Case 2.2.1.1.0

In Case 2.2.2.1.1.0, there is no westbound vehicle in the entire coverage of the eastbound cluster, as shown in Fig. 7. This case happens when the length of the eastbound cluster satisfies the following condition:

$$
l_{e}<E\left[d_{c}^{w}\right]-2 R_{v}
$$

and the probability that Case 2.2.2.1.1.0 happens is given by

$$
p_{222110}=\operatorname{Pr}\left\{l_{e}<E\left[d_{c}^{w}\right]-2 R_{v}\right\}
$$

According to [7], the average distance between two neighbor eastbound clusters is

$$
E\left[d_{c}^{w}\right]=R_{v}+1 / \lambda_{w}
$$

Obviously, given $E\left[d_{c}^{w}\right]$ and $E\left[d_{v}^{e}\right]$, we can obtain

$$
p_{222110}=\operatorname{Pr}\left\{l_{e}<E\left[d_{c}^{w}-2 R_{v}\right]\right\}=1-\left(1-e^{-\lambda_{e} R_{v}}\right)^{k_{222110}},
$$

where $k_{222110}=\left(E\left[d_{c}^{w}\right]-2 R_{v}\right) / E\left[d_{v}^{e}\right]$.

In this case, node 2 will store and carry the information until it meets a westbound vehicle, when it will 
forward the information to the westbound vehicle, which will further forward the information to RSU1. Since the westbound vehicle closest to node 2 is at least $x+0.5 E\left[d_{c}^{w}\right]$ away from RSU1, the delivery delay can be approximately calculated as

$$
T_{222110}=\left(x+0.5 E\left[d_{c}^{w}\right]\right) / v_{w} .
$$

Case 2.2.2.1.1.1

In Case 2.2.2.1.1.1, there is one or more westbound vehicles within the communication range of a node other than node 2 in the eastbound cluster. Obviously, this case happens with probability

$$
p_{222111}=1-p_{222110} .
$$

In this case, node 2 will directly transmit the information to a westbound node, which will further forward the information to RSU1. According to [7], the average length of an eastbound cluster is

$$
E\left[l_{e}\right]=\left(e^{\lambda_{e} R_{v}}-1\right) \cdot\left[1 / \lambda_{e}-R_{v} \cdot e^{-\lambda_{e} R_{v}} /\left(1-e^{-\lambda_{e} R_{v}}\right)\right] .
$$

Since the closest westbound vehicle to node 2 is at most $x+0.5 E\left[l_{e}\right]$ away from RSU1, the delivery delay can be approximately calculated as

$$
T_{222111}=\left(x+0.5 E\left[l_{e}\right]\right) / v_{w} .
$$

Considering all the above cases, we have

$$
T_{R S U 1}=\left[p_{22210} \cdot p_{222100} \cdot T_{222100}+p_{22211} \cdot\left(p_{222111} \cdot T_{222111}+p_{222110} \cdot T_{222110}\right)\right] \cdot p_{2221} \cdot
$$

\subsubsection{Analysis of $T_{R S U 2}$}

Similar to the analysis of $T_{R S U 1}$, we assume that the source node is statistically located in the center of its cluster. As we assumed that the source node is an eastbound vehicle, the forwarding direction of the incident information to RSU2 is the same as the moving direction of the source node. Since the direct transmission delay can be ignored, the incident information can be delivered to the first vehicle in the cluster without any delay. In this case, the first vehicle becomes a forwarding node, which is denoted by node 4, as shown in Fig. 9. If node 4 is in the communication radius of RSU2, the information can be transmitted to RSU2 directly. Thus, we only need to consider the case where node 4 is out of the communication radius of RSU2. This case happens if the length of the eastbound cluster satisfies the following condition:

$$
l_{e} / 2-R_{v}<d-x-R_{u}
$$

and the probability that this case happens is given by

$$
p_{2222}=\operatorname{Pr}\left\{l_{e} / 2-R_{v}<d-x-R_{u}\right\} .
$$

Similar to Eq.(9), $p_{2222}$ can be calculated as follows 


$$
p_{2222}=\operatorname{Pr}\left\{l_{e} / 2-R_{v}<d-x-R_{u}\right\}=1-\left(1-e^{-\lambda_{e} R_{v}}\right)^{k_{2222}},
$$

where $k_{2222}=2\left(d-x-R_{u}+R_{v}\right) / E\left[d_{v}^{e}\right]$.

Let $E_{2222}$ denote the conditional expectation of $l_{e} / 2$ under condition $l_{e} / 2<d-x-R_{u}+R_{v}$. We can obtain

$$
E_{2222}=E\left[l_{e} / 2 \mid l_{e} / 2<d-x-R_{u}+R_{v}\right]=0.5 E\left[d_{v}^{e}\right] E\left[n_{e} \mid n_{e}<k_{2222}\right],
$$

where $E\left[n_{e} \mid n_{e}<k_{2222}\right]=\left[1-\left(k_{2222}+1\right)\left(1-e^{-\lambda_{e} R_{v}}\right)^{k_{2222}}+k_{2222}\left(1-e^{-\lambda_{e} R_{v}}\right)^{k_{2222}+1}\right] /\left\{\left[1-\left(1-e^{-\lambda_{e} R_{v}}\right)^{k_{2222}}\right] e^{-\lambda_{e} R_{v}}\right\}$.

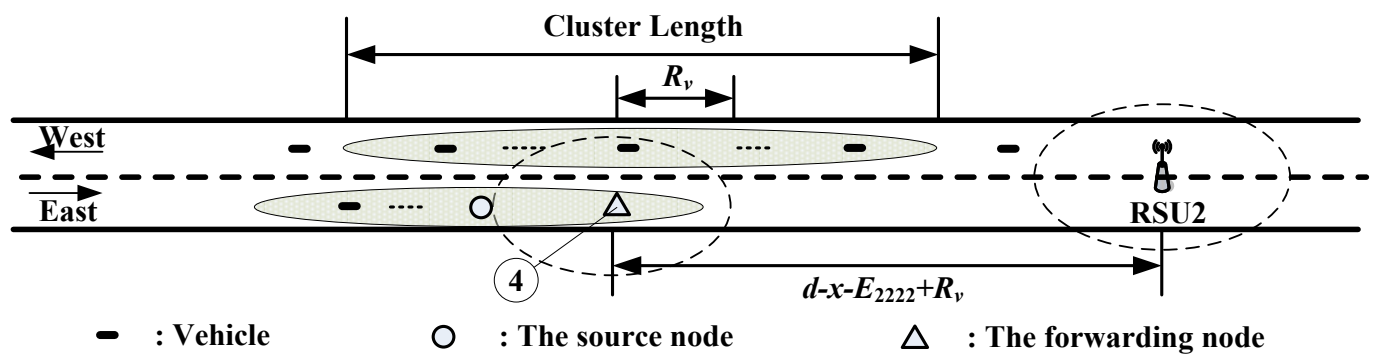

Fig.9 The westbound cluster is out of the communication radius of RSU2.

Similarly to the analysis of $T_{R S U 1}$, this case can further be divided into two sub-cases.

- Case 2.2.2.2.0: There are one or more westbound vehicles in the coverage of node 4 .

- Case 2.2.2.2.1: There is no westbound vehicle in the coverage of node 4.

Case 2.2.2.2.0

Case 2.2.2.2.0 happens when there is at least one westbound vehicle within the communication radius of node 4. Let $p_{22220}$ denote the probability that Case 2.2.2.2.0 happens. Thus, we have

$$
p_{22220}=1-e^{-2 \lambda_{w} R_{v}} .
$$

In this case, if the last vehicle of the westbound cluster is within the communication radius of RSU2, the information can be transmitted to RSU2 directly and the direct transmission delay can be ignored. However, if the last vehicle of the westbound cluster is out of the communication radius of RSU2, i.e., the length of the westbound cluster satisfies the following condition:

$$
l_{w} / 2<d-x-E_{2222}+R_{v},
$$

the information will be forwarded to RSU2 by node 4 . The probability of this case is given by

$$
p_{222200}=\operatorname{Pr}\left\{l_{w} / 2<d-x-E_{2222}+R_{v}\right\} .
$$

Similar to Eq.(15), we can obtain

$$
p_{222200}=\operatorname{Pr}\left\{l_{w} / 2<d-x-E_{2222}+R_{v}\right\}=1-\left(1-e^{-\lambda_{w} R_{v}}\right)^{k_{222200}},
$$

where $k_{222200}=2\left(d-x-E_{2222}+R_{v}\right) / E\left[d_{v}^{w}\right] .$. Therefore, the delivery delay can be calculated as 


$$
T_{222200}=\left(d-x-R_{u}-E_{2222}+R_{v}\right) / v_{e} .
$$

Case 2.2.2.2.1

Case 2.2.2.2.1 happens when there is no westbound vehicle within the communication radius of node 4 . The probability that Case 2.2.2.2.1 happens can be calculated as

$$
p_{2221}=1-p_{22220} \text {. }
$$

In this case, the information will be forwarded to RSU2 by node 4 . Thus, the delivery delay $T_{22221}$ equals to $T_{222200 .}$

Considering all the above cases, we have

$$
\begin{gathered}
T_{R S U 2}=p_{2222} \cdot\left(p_{22220} \cdot p_{222200} \cdot T_{222200}+p_{22221} \cdot T_{22221}\right) . \\
T_{S 2}=\max \left\{T_{R S U 1}, T_{R S U 2}\right\} .
\end{gathered}
$$

2.3 Case 2.3: The incident occurs at $x \in\left[d-R_{v}, d\right]$

In Case 2.3, the incident occurs within the communication radius of RSU2. After the source node collects the information, it can transmit the incident information to RSU2 directly. Thus, the time it takes for the source node to directly forward the incident information to RSU2 can be ignored. Let $f_{3}(x)$ denote the conditional probability density function of the distance $x$ in $\left[d-R_{v}, d\right]$. Then, the average information delivery delay can be written as follows

$$
T_{3}=T_{03}+T_{S 3},
$$

where $T_{03}$ is the average time that it takes for the source node to arrive at the occurring location of the incident information, and $T_{S 3}$ is the average time that it takes for the source node to deliver the incident information to RSU1. Next we analyze $T_{03}$ and $T_{S 3}$, respectively.

\subsubsection{Analysis of $T_{03}$}

Similar to the analysis of $T_{01}, T_{03}$ can be calculated as

$$
T_{03}=1 / v_{e} \cdot\left(\int_{d-R_{v}}^{d} x \cdot f_{3}(x) d x-d+R_{v}\right)+1 /\left(\lambda_{e} v_{e}\right) .
$$

\subsubsection{Analysis of $T_{S 3}$}

Similar to the analysis of $T_{R S U 1}$, we assume that the source node is statistically located in the center of its cluster, as shown in Fig. 10. As we assumed that the source node is an eastbound vehicle, the delivery direction of the incident information to RSU1 is opposite to the moving direction of the source node. Since the direct transmission delay is ignored, the incident information can be delivered to the last vehicle in the cluster without any delay. In this case, this vehicle becomes a forwarding node, which is denoted by node 5 in Fig. 10. If node 5 is 
in the communication radius of RSU1, the information can be transmitted to RSU1 directly. Thus, we only need to consider the case where node 5 is out of the communication radius of RSU1. This case happens if the length of the eastbound cluster satisfies the following condition:

$$
l_{e} / 2-R_{v}<x-R_{u}
$$

and the probability that this case happens is given by

$$
p_{232}=\operatorname{Pr}\left\{l_{e} / 2-R_{v}<x-R_{u}\right\} .
$$

Similar to Eq.(9), we can obtain

$$
p_{232}=\operatorname{Pr}\left\{l_{e} / 2-R_{v}<x-R_{u}\right\}=1-\left(1-e^{-\lambda_{e} R_{v}}\right)^{k_{232}},
$$

where $k_{232}=2\left(x-R_{u}+R_{v}\right) / E\left[d_{v}^{e}\right]$.

Let $E_{232}$ denote the conditional expectation of $l_{e} / 2$ under condition $l_{e} / 2<x-R_{u}+R_{v}$. We can obtain

$$
\begin{aligned}
E_{232} & =E\left[l_{e} / 2 \mid l_{e} / 2<x-R_{u}+R_{v}\right] \\
& =0.5 E\left[d_{v}^{e}\right] \cdot\left[1-\left(k_{232}+1\right)\left(1-e^{-\lambda_{e} R_{v}}\right)^{k_{232}}+k_{232}\left(1-e^{-\lambda_{e} R_{v}}\right)^{k_{232}+1}\right] /\left\{\left[1-\left(1-e^{-\lambda_{e} R_{v}}\right)^{k_{232}}\right] e^{-\lambda_{e} R_{v}}\right\}
\end{aligned}
$$

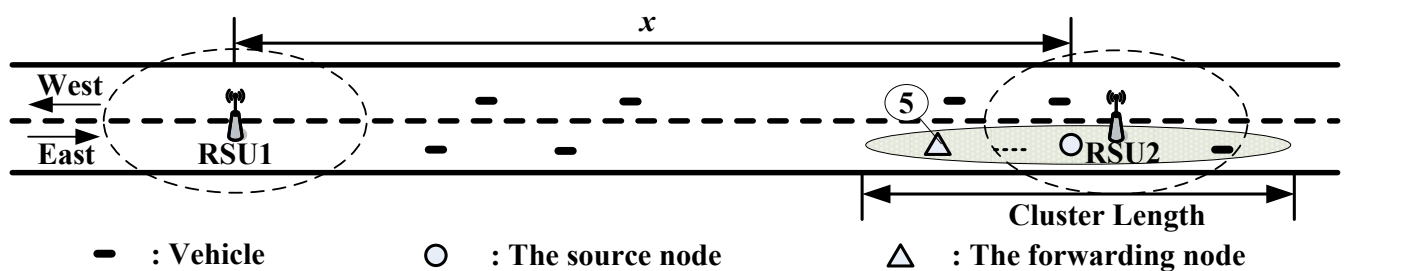

Fig.10 The eastbound forwarding node is out of the communication radius of RSU1

Further, we divide this case into two sub-cases:

- Case 2.3.2.0: There are one or more westbound vehicles within the coverage of node 5.

- Case 2.3.2.1: There is no westbound vehicle in the coverage of node 5.

Case 2.3.2.0

Case 2.3.2.0 happens when there is at least one westbound vehicle within the communication radius of node 5. Let $p_{2320}$ denote the probability that Case 2.3.2.0 happens. Thus, we have

$$
p_{2320}=1-e^{-2 \lambda_{w} R_{v}}
$$

In this case, the first vehicle of the corresponding westbound cluster becomes a forwarding node, which is denoted by node 6 , as shown in Fig. 11. If node 6 is within the communication radius of RSU1, the information can be directly transmitted to RSU1 and the direct transmission delay can be ignored. Thus, we only need to analyze that the case where node 6 is out of the communication range of RSU1, i.e., the length of the westbound cluster satisfies the following condition: 


$$
l_{w} / 2<x-E_{232}+R_{v} .
$$

In this case, node 6 will store and carry the information until it enters the communication radius of RSU1, when it will transmit the information to RSU1 directly.

Let $p_{23200}$ denote the probability that this case happens. Thus, we have

$$
p_{23200}=\operatorname{Pr}\left\{l_{w} / 2<x-E_{232}+R_{v}\right\} .
$$

Similar to Eq.(15), we can obtain

$$
p_{23200}=\operatorname{Pr}\left\{l_{w} / 2<x-E_{232}+R_{v}\right\}=1-\left(1-e^{-\lambda_{w} R_{v}}\right)^{k_{23200}},
$$

where $k_{23200}=2\left(x-E_{232}+R_{v}\right) / E\left[d_{v}^{w}\right] .$.

Therefore, the delivery delay for node 6 to forward the incident information to RSU1 can be approximately calculated as

$$
T_{23200}=\left(R_{v}+x-E_{232}-0.5 E\left[l_{w} / 2 \mid l_{w} / 2<x-E_{232}+R_{v}\right]\right) / v_{w},
$$

where

$$
\begin{aligned}
& E\left[l_{w} / 2 \mid l_{w} / 2<x-E_{232}+R_{v}\right] \\
& =0.5 E\left[d_{v}^{w}\right] \cdot\left[1-\left(k_{23200}+1\right)\left(1-e^{-\lambda_{w} R_{v}}\right)^{k_{23200}}+k_{23200}\left(1-e^{-\lambda_{w} R_{v}}\right)^{k_{23200}+1}\right] /\left\{\left[1-\left(1-e^{-\lambda_{w} R_{v}}\right)^{k_{23200}}\right] e^{-\lambda_{w} R_{v}}\right\}
\end{aligned}
$$

Fig.11 The westbound forwarding node is out of the communication radius of RSU1.

Case 2.3.2.1

Case 2.3.2.1 happens when there is no westbound vehicle within the communication radius of node 5. The probability that case 2.3.2.1 happens can be calculated as

$$
p_{2321}=1-p_{2320} .
$$

In this case, RSU2 should store and deliver the incident information to the upcoming western vehicles which will forward the incident information to RSU1. Then, the delivery delay can be approximately calculated as follows

$$
T_{2321}=\left(d-R_{v}\right) / v_{w}+1 /\left(\lambda_{w} v_{w}\right) .
$$

Considering all the above cases, we have

$$
T_{S 3}=\left(p_{2320} \cdot p_{23200} \cdot T_{23200}+p_{2321} \cdot T_{2321}\right) \cdot p_{232} .
$$

The average information delivery delay can be calculated as follows 


$$
T=\int_{0}^{R_{v}} T_{1} \cdot f_{1}(x) d x+\int_{R_{v}}^{d-R_{v}} T_{2} \cdot f_{2}(x) d x+\int_{d-R_{v}}^{d} T_{3} \cdot f_{3}(x) d x .
$$

\section{Simulation Results and Discussion}

In this section, we verify the effectiveness of the mathematical model derived in Section III through simulation results and investigate the impacts of different parameters on the average delivery delay, respectively. The simulation experiments were conducted using MATLAB. In the simulation experiments, we assumed that the location of an incident is uniformly distributed in interval $[0, d]$, and the transmission radius of a vehicle is $250 \mathrm{~m}$, i.e., $R_{v}=250 \mathrm{~m}$, which follows the US Federal Communications Commission (FCC) regulations. Each simulation result is an average over 1000 simulations.

Fig. 12 shows the impact of the communication radius of an RSU on the average delivery delay. It is seen that the simulation results are very close to the analytical results, which means the mathematical model is effective. Moreover, the communication radius of an RSU has little impact on the average delivery delay. This is because the incident information is delivered from a vehicle to an RSU, not an RSU to a vehicle, and thus the communication radius of a vehicle has a larger impact on the delivery delay. Therefore, without loss of generality, we assume $R_{u}=250 \mathrm{~m}$ in the following simulation experiments.

Fig. 13 - Fig. 15 show the average delivery delay under different traffic loads. Fig. 16 - Fig. 18 show the average delivery delay under different vehicle speeds. It is seen that simulation results are very close to the analytical results. On the other hand, the average delivery delay decreases with the increase of the vehicle speed and the increase of the traffic arrival rate, respectively. Moreover, the impact of the vehicle speed on the average delivery delay is much larger than that of the traffic arrival rate. This is because that a sparse network with a low traffic arrival rate has a small probability to form a large cluster which can help reduce the delivery delay. A higher speed may enable a forwarding node to enter the communication range of an RSU more rapidly and thus more significantly reduce the average delivery delay.

Fig. 19 shows the average delivery delay under different distances between two neighbor RSUs. It is seen that the simulation results are very close to the analytical results. Moreover, with an increasing distance between two neighbor RSUs, the average delivery delay decreases. Therefore, the mathematical model can be used to estimate the average delivery delay given a distance between two neighbor RSUs, which can provide a reference basis for RSU deployment in order to meet the delay requirement of some applications. 


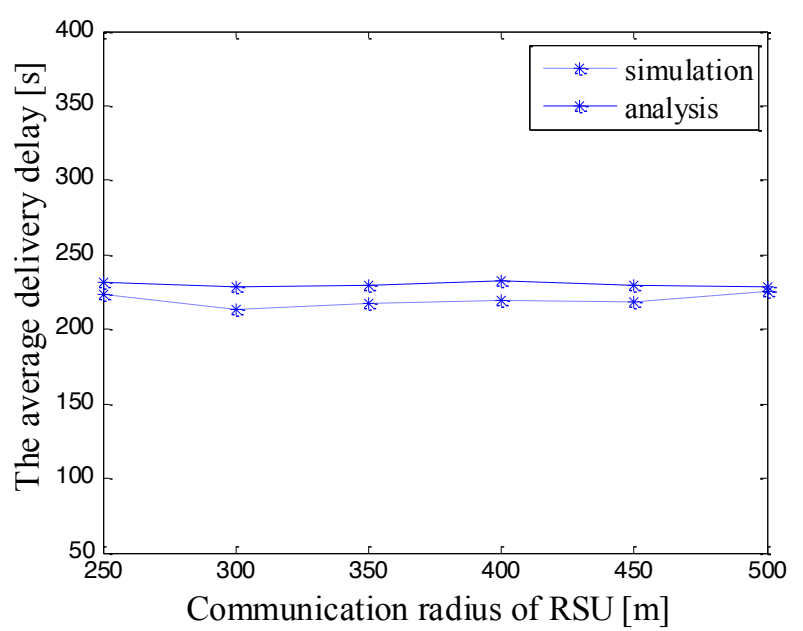

Fig. 12. Impact of $R_{u}$ on the average delivery delay $\left(d=10000 \mathrm{~m}, R_{v}=250 \mathrm{~m}, v_{w}=30 \mathrm{~m} / \mathrm{s}, v_{e}=40 \mathrm{~m} / \mathrm{s}, \lambda_{w}=\lambda_{e}=0.0030 \mathrm{veh} / \mathrm{m}\right)$.

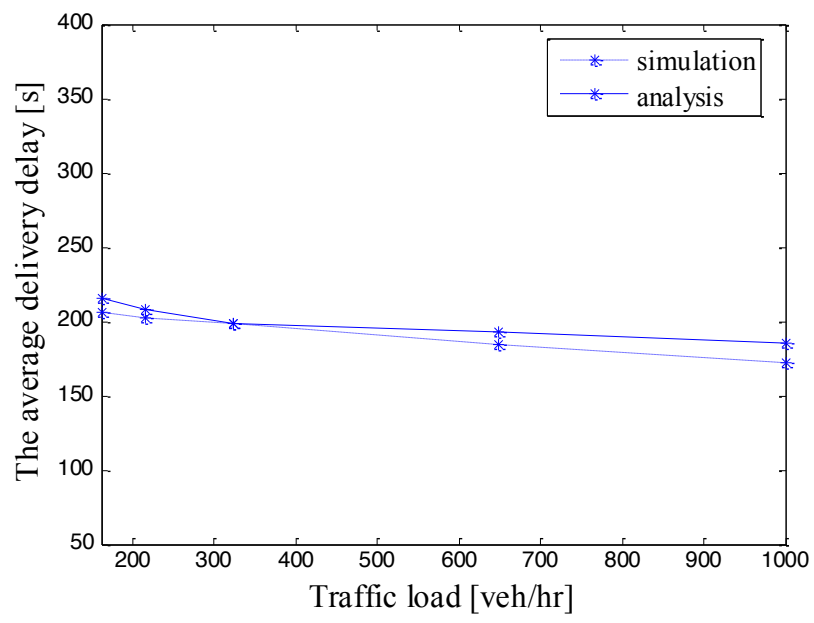

Fig. 13. Average delivery delay vs traffic load $\left(d=10000 \mathrm{~m}, R_{u}=R_{v}=250 \mathrm{~m}, v_{w}=v_{e}=40 \mathrm{~m} / \mathrm{s}, \lambda_{w}=\lambda_{e}\right)$.

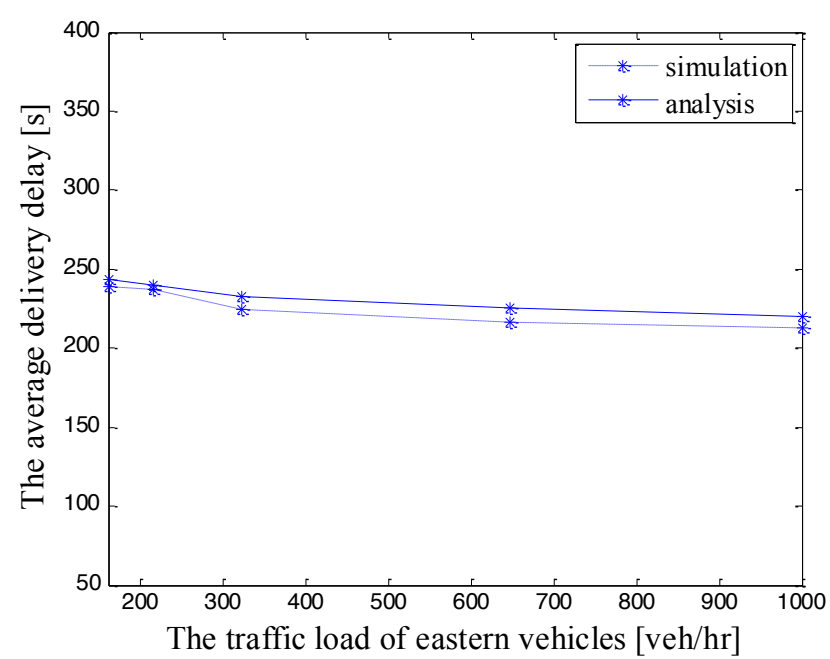

Fig. 14. Average delivery delay vs traffic load of eastern vehicles ( $\left.d=10000 \mathrm{~m}, R_{u}=R_{v}=250 \mathrm{~m}, v_{w}=30 \mathrm{~m} / \mathrm{s}, v_{e}=40 \mathrm{~m} / \mathrm{s}, \lambda_{w}=0.003 \mathrm{veh} / \mathrm{m}\right)$. 


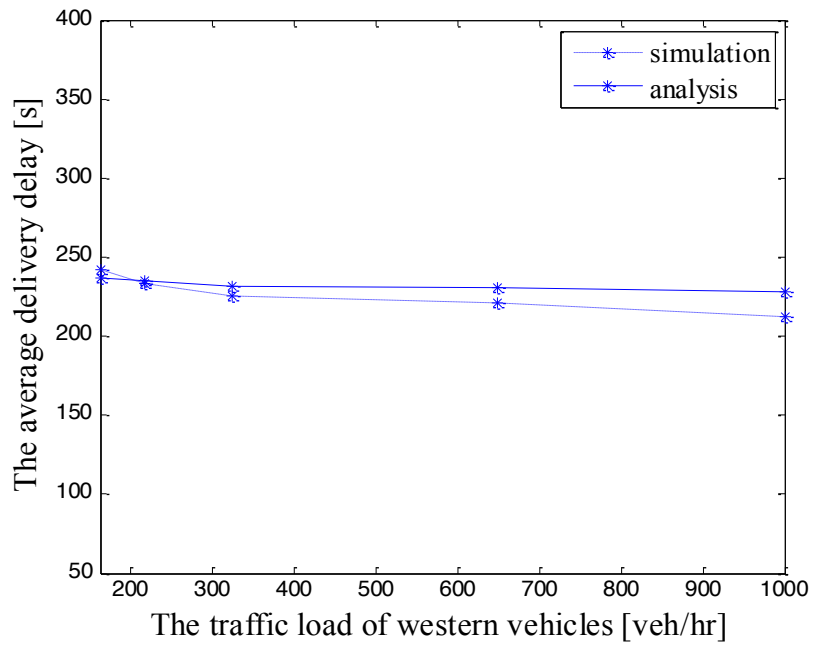

Fig. 15. Average delivery delay vs traffic load of western vehicles $\left(d=10000 \mathrm{~m}, R_{u}=R_{v}=250 \mathrm{~m}, v_{w}=30 \mathrm{~m} / \mathrm{s}, v_{e}=40 \mathrm{~m} / \mathrm{s}, \lambda_{e}=0.003 \mathrm{veh} / \mathrm{m}\right)$.

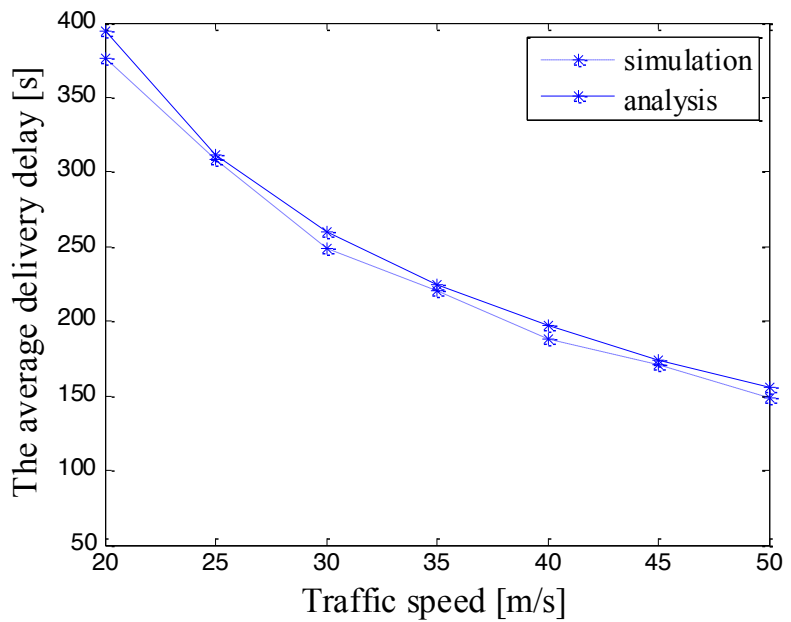

Fig. 16. Average delivery delay vs traffic speed ( $\left.d=10000 \mathrm{~m}, R_{u}=R_{v}=250 \mathrm{~m}, v_{w}=v_{e}, \lambda_{w}=\lambda_{e}=0.003 \mathrm{veh} / \mathrm{m}\right)$.

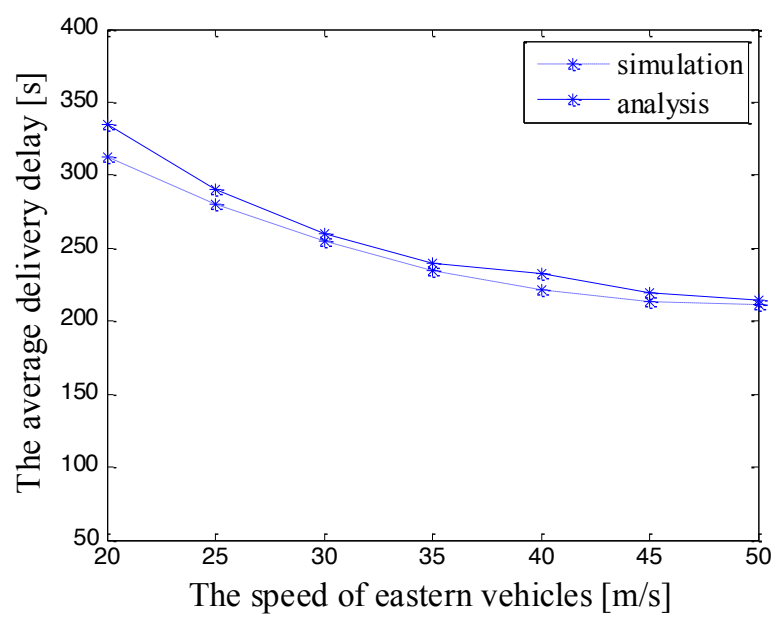

Fig. 17. Average delivery delay vs the speed of eastern vehicles $\left(d=10000 \mathrm{~m}, R_{u}=R_{v}=250 \mathrm{~m}, v_{w}=30 \mathrm{~m} / \mathrm{s}, \lambda_{w}=\lambda_{e}=0.003 \mathrm{veh} / \mathrm{m}\right)$. 


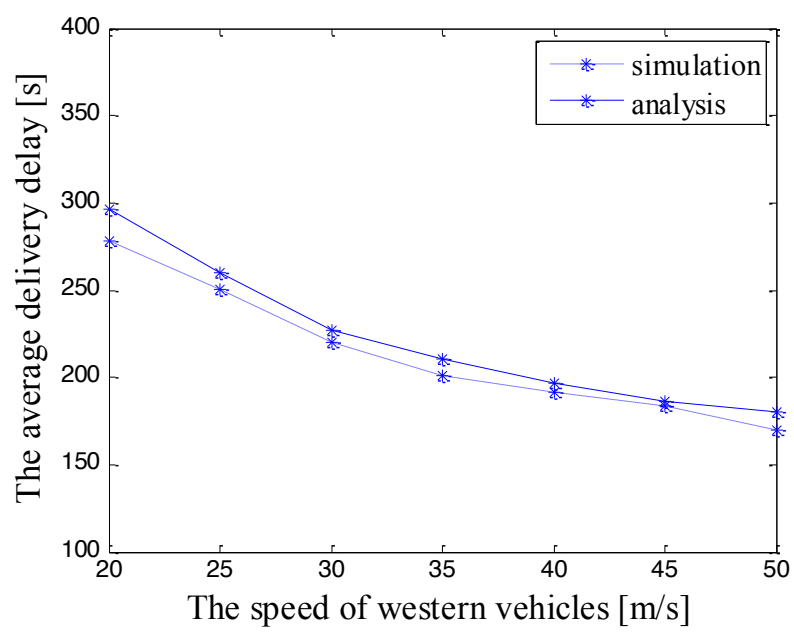

Fig. 18. Average delivery delay vs the speed of eastern vehicles $\left(d=10000 \mathrm{~m}, R_{u}=R_{v}=250 \mathrm{~m}, v_{e}=40 \mathrm{~m} / \mathrm{s}, \lambda_{w}=\lambda_{e}=0.003 \mathrm{veh} / \mathrm{m}\right)$.

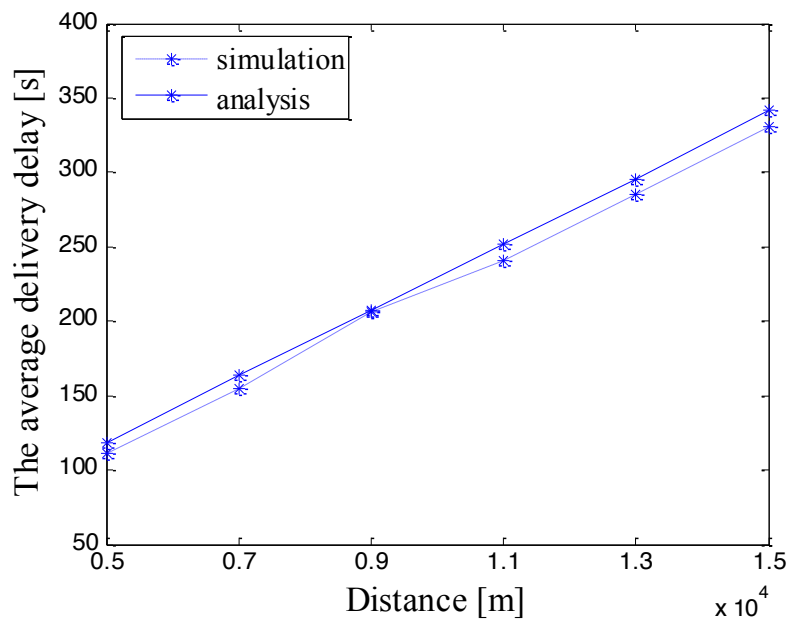

Fig. 19. Average delivery delay vs distance between RSUs $\left(R_{u}=R_{v}=250 \mathrm{~m}, v_{w}=30 \mathrm{~m} / \mathrm{s}, v_{e}=40 \mathrm{~m} / \mathrm{s}, \lambda_{w}=\lambda_{e}=0.003 \mathrm{veh} / \mathrm{m}\right)$.

\section{Conclusion}

In this paper, we developed a mathematical model for analyzing information delivery delay in a straight highway scenario where two RSUs are deployed at a distance without any direct connection and vehicles are sparsely distributed with road condition information randomly generated between the two neighbor RSUs. The mathematical model takes into account the vehicle speed, the vehicle density, the likelihood of an incident, and the distance between two RSUs, and its effectiveness is verified through simulation results. Given the delay constraint of time-critical applications, this model can be used to estimate the maximum distance allowed between two neighbor RSUs, which can provide a reference for the deployment of RSUs in such scenarios. 


\section{References}

[1] M. J. Khabbaz, H. M. Alazemi, and C. M. Assi, "Modeling and delay analysis of a retransmission-based bundle delivery scheme for intermittent roadside communication networks," Intelligent Transportation Systems, IEEE Transactions on, vol. 14, no. 2, June 2013, pp. 700-708.

[2] M. J. Khabbaz, W. F. Fawaz, and C. M. Assi, "Modeling and Delay Analysis of Intermittently Connected Roadside Communication Networks," Vehicular Technology, IEEE Transactions on, vol. 61, no. 6, July 2012, pp. 2698-2706.

[3] M. J. Khabbaz, W. F. Fawaz, and C. M. Assi, "A simple free-flow traffic model for vehicular intermittently connected networks," Intelligent Transportation Systems, IEEE Transactions on, vol. 13, no. 3, Sept. 2012, pp. 1312-1326.

[4] M. J. Khabbaz, W. F. Fawaz, and C. M. Assi, "Which vehicle to select," Communications Letters, IEEE, vol. 16, no. 6, June 2012, pp. 812-815.

[5] A. Abdrabou and W. Zhuang, "Probabilistic delay control and road side unit placement for vehicular ad hoc networks with disrupted connectivity," Selected Areas in Communications, IEEE Journal on, vol. 29, no. 1, Jan. 2011, pp. 129-139.

[6] A. Abdrabou, B. Liang, and W. Zhuang, "Delay Analysis for Sparse Vehicular Sensor Networks with Reliability Considerations," Wireless Communications, IEEE Transactions on, vol. 12, no. 9, Aug. 2013, pp. $4402-4413$.

[7] N. Wisitpongphan, F. Bai, P. Mudalige, V. Sadekar, and O. Tonguz, "Routing in sparse vehicular ad hoc wireless networks," Selected Areas in Communications, IEEE Journal on, vol. 25, no. 8, Oct. 2007, pp. 1538-1556.

[8] A. B. Reis, S. Sargento, and O. K. Tonguz, "On the performance of sparse vehicular networks with road side units," in Vehicular Technology Conference (VTC Spring), 2011 IEEE 73rd, Yokohama, Japan, May 2011, pp. 1-5.

\section{ACKNOWLEDGMENT}

This work was supported by the National Natural Science Foundation of China under Grant No. 61372105 and the Six Talent Peaks Project in Jiangsu Province under Grant No. DZXX-010. 


\section{BIOGRAPHY}

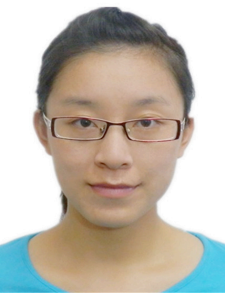

optimization.

Yu Wang received her M.S. degree in the School of Science from Yanshan University, Qinhuangdao, China, in 2011. She is currently studying towards her Ph.D degree in the School of Information Science and Engineering at Southeast University, Nanjing, China. Her research interests is in the area of vehicular ad hoc networks, focusing on roadside unit deployment and

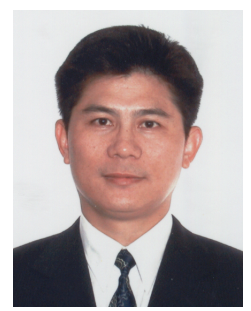

Jun Zheng is a Full Professor with the School of Information Science and Engineering at Southeast University (SEU), China. He received his $\mathrm{Ph}$. D. degree in electrical and electronic engineering from The University of Hong Kong in 2000. Before joining SEU in 2008, he was with the School of Information Technology and Engineering, University of Ottawa, Canada.

He has co-authored (first author) the book Wireless Sensor Networks: A Networking Perspective, and the book Optical WDM Networks: Concepts and Design Principle, both published by Wiley-IEEE Press. He has co-authored over 100 technical papers in refereed journals and peer-reviewed conference proceedings. He is the co-recipient of an ICC 2014 Best Paper Award. His current research interests include vehicular ad hoc networks, mobile communication networks, wireless sensor networks, focused on network architectures and protocols.

He serves as a Technical Editor of IEEE Communications Magazine and Editor in Chief of EAI Endorsed Transactions on Mobile Communications and Applications. He is also an editorial board member of several other refereed journals, including Elsevier Ad Hoc Networks Journal and Springer Wireless Networks. He has co-edited 12 special issues for different refereed journals and magazines, including IEEE Communications Magazine, IEEE Network, and IEEE Journal on Selected Areas in Communications, all as Lead Guest Editor. He has served as the founding General Chair of AdHocNets'09, General Chair of AccessNets'07, and TPC or Symposium Co-Chair for many international conferences and symposia, including IEEE ICC $(2009,2011,2015)$ and GLOBECOM $(2008,2010,2012)$. He has also served as a technical program committee member for a number of international conferences and symposiums. He is a senior member of the IEEE, IEEE Communications Society, and IEEE Vehicular Technology Society.

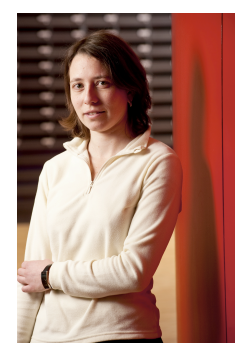

Nathalie Mitton received the MSc and PhD. degrees in Computer Science from INSALyon in 2003 and 2006 respectively. She received her Habilitation à diriger des recherches (HDR) in 2011 
from Université Lille 1. She is currently an Inria full researcher since 2006 and from 2012, she is the scientific head of the Inria FUN team which is focused on small computing deviceslike electronic tags and sensor networks. Her research interests are mainly focused on self-organization, self-stabilization, energy efficient routing and neighbor discovery algorithms for wireless sensor networks as well as RFID middlewares. She is involved in the set up of the FIT platforms (http://fit-equipex.fr/), the FP7 Aspire or VITAL projects and in several program and organization committees such as AdHocNets 2015 and 2014, AdHocnow 2015, HPCC 2014, WiMob 2013, MASS 2012 \& 2011, LoGASN 2012 , WPMC2012, IST-AWSN 2012, iThings 2012, Comnet-iot 2012, etc. She also supervises several PhD students and engineers 